\title{
Genetic Variability in Slovenian Cohort of Patients with Oculocutaneous Albinism
}

\author{
Tinka Hovnik, ${ }^{1,2, \star}$ Maruša Debeljak, ${ }^{1}$ Manca Tekavčič Pompe, ${ }^{2,3}$ Sara Bertok, ${ }^{4}$ \\ Tadej Battelino, ${ }^{2,4}$ Branka Stirn Kranjc ${ }^{2,3}$ and Katarina Trebušak Podkrajšek ${ }^{1,2}$ \\ ${ }^{1}$ University Medical Centre Ljubljana, University Children's Hospital, Clinical Institute for Special Laboratory Diagnostics, \\ Vrazov trg 1, SI-1000 Ljubljana, Slovenia \\ ${ }^{2}$ University of Ljubljana, Faculty of Medicine, Vrazov trg 2, SI-1000 Ljubljana, Slovenia \\ ${ }^{3}$ University Medical Centre Ljubljana, Eye Hospital, Grablovičeva 46, SI-1000 Ljubljana, Slovenia \\ ${ }^{4}$ University Medical Centre Ljubljana, University Children's Hospital, Department of Pediatric Endocrinology, Diabetes and \\ Metabolic Diseases, Bohoričeva 20, SI-1000 Ljubljana, Slovenia \\ *Corresponding author: E-mail: tinka.hovnik@kclj.si
}

Received: 04-23-2021

\begin{abstract}
Oculocutaneous albinism (OCA) is an inherited disorder affecting the visual system and skin pigmentation. Our aim was to evaluate genetic and clinical heterogeneity in a cohort of Slovenian paediatric patients with clinically suspected OCA using advanced molecular-genetics approach. In as much as 20 out of 25 patients, genetic variants explaining their clinical phenotype were identified. The great majority of patients (15/25) had genetic variants in TYR gene associated with OCA type 1, followed by variants in TYRP1, SLC45A2 and HPS1 genes causative for OCA3, OCA4 and Hermansky-Pudlak syndrome type 1, respectively. We concluded that OCA phenotype could not predict genotype and vice versa. Nevertheless, the diagnostic yield after targeted next generation sequencing (NGS) was $80 \%$ and proved to be affective in our paediatric cohort of patients with various degree of OCA. Even in 16 patients with normal complexion the diagnostic yield was $62,5 \%$. Interestingly, we have identified a patient of white European ancestry with OCA3, which is an extremely rare report, and one patient with OCA due to the Hermansky-Pudlak syndrome type 1.
\end{abstract}

Keywords: Oculocutaneous albinism, Hermansky-Pudlak syndrome type 1, next generation sequencing, genetic variant

\section{Introduction}

Albinism is a rare inherited disorder affecting visual system and skin pigmentation, and has a global incidence of approximately 1 in $17.000 .^{1,2}$ The most handicapping manifestations are ocular abnormalities, namely reduced visual acuity with nystagmus, strabismus, photophobia, foveal hypoplasia and misrouting of optic nerve fibres at the chiasm. ${ }^{3,4}$ Albinism is clinically classified in three groups. In ocular albinism (OA, OMIM \# 300500) pigmentation is impaired only in the eyes, while in oculocutaneous albinism (OCA) also pigmentation of the skin and/ or hair is impaired. In syndromic forms, such as syndrome Hermansky-Pudlak and Chediak-Higashi syndrome, additional manifestations are present. ${ }^{5}$

OCA is associated with defective biosynthesis or transport of melanin. ${ }^{6}$ Each of the several OCA types is related to the individual genetic defect. OCA 1 is caused by disease causing variants in TYR gene $\left(\mathrm{OMIM}^{*} 606933\right)^{7}$ encoding enzyme tyrosinase catalysing the first two steps in melanin biosynthesis. ${ }^{8}$ In OCA1A subtype, enzyme activity is completely abolished and patients have no pigment and severe ocular symptoms. In OCA1B subtype, residual tyrosinase activity is present and consequently patients may develop some pigment after infancy. ${ }^{9}$ Among patients with mild OCA1B, two relatively common TYR variants, namely NM_000372.4:c.575C >A (p.Ser192Tyr) and NM_000372.4: c.1205G>A (p.Arg402Gln) located in cis and were reported as a prevalent cause when inherited in trans with pathogenic TYR variant. ${ }^{10-14}$

OCA2 is the commonest type and represents about $30 \%$ of OCA worldwide. ${ }^{15}$ It is caused by disease causing variants in OCA2 gene (OMIM* 611409) encoding inte- 
gral melanosomal transmembrane protein. ${ }^{16}$ Patients have various amounts of cutaneous pigment. OCA3 is more common in Africa but is extremely rare in white European or Asiatic populations. ${ }^{17}$ It is caused by disease causing variants in TYRP1 gene (OMIM* 115501) encoding tyrosinase-related protein 1 involved in melanin biosynthesis pathway. OCA4 is caused by disease causing variants in SLC45A2 gene $\left(\mathrm{OMIM}^{\star} 606202\right)^{18}$ encoding melanosomal MATP protein, that might affects tyrosinase activity through the regulation of the melanosomal $\mathrm{pH} .{ }^{19}$ In 2013 , three additional rare OCA types, namely OCA5, OCA6 and OCA7, and their associated genes, were added to the consensus list of albinism types. ${ }^{2}$ Recently, additional gene was reported in relation to the oculocutaneous albinism, namely dopachrome tautomerase gene $(D C T)$ shown to be associated with autosomal recessive form. ${ }^{20}$

Clinical evaluation of a child with suspected albinism should consist of full clinical examination with special emphasis on nystagmus evaluation, iris transillumination defects detection, foveal hypoplasia gradation and retinal pigment epithelium evaluation. ${ }^{21}$ The diagnostic tools of great importance are optical coherence tomography (OCT) for foveal hypoplasia gradation ${ }^{22}$ and visual evoked potentials (VEP) which can demonstrate misrouting of optic nerve fibers in the chiasm. ${ }^{23}$ Despite significant technological advances in genetic testing, a substantial fraction of individuals with OCA remains genetically unexplained. Missing heritability after common four gene testing was reported to be between 10 and $25 \%$ in complete OCA and up to $50 \%$ in partial $\mathrm{OCA}^{24}$ and was still as high as $28 \%$ in panel based NGS approach. ${ }^{12}$ Nevertheless, next generation sequencing (NGS) based genetic testing presents a possibility for an early definitive diagnosis and management of the disease, especially since OCA types are often difficult to differentiate clinically. Our aim was to evaluate genetic and clinical heterogeneity in a cohort of Slovenian paediatric patients with clinically suspected OCA using combined molecular-genetic approach.

\section{Methods}

\section{1. Patients}

Paediatric individuals with clinical signs of OCA were examined at the outpatient clinic of the Eye Hospital at the University Medical Centre Ljubljana. Clinical ophthalmological examination with visual acuity for distance and near, colour vision, perimetry, ocular motility, biomicroscopy, fundus examination andelectrophysiology was performed. Main albinism signs in the studied group of children were: ocular hypopigmentation with iris translucency or fundus hypopigmentation, foveal hypoplasia, misrouting, nystagmus, skin and hair hypopigmentation. Best corrected monocular and binocular visual acuity was tested using Teller Acuity Cards, Cambridge Acuity Crowding Cards, Lea Symbols Cards; verbal optotypes for distance and near visual acuity were tested with HOTV optotype and Jaeger tables. Colour vision was assessed at first or follow-up examinations with non-verbal and verbal Ishihara plates. Visual evoked potentials (VEPs) to flash and onset stimulation were recorded in all children ${ }^{25}$ at presentation and follow-up, and macular OCT (spectral domain optical coherence tomography) in some cooperative children. OCT images, were obtained in mydriasis with OCT Topcon 3D OCT-1000 (Topcon Medical Systems, Tokyo, Japan) and/or Spectralis HRA + OCT (Heidelberg Engineering, Germany). The retinal thickness and total macular volume were determined using the OCT apparatus software.

All participants or parents of minors gave their written informed consent prior to the study (approved by the Republic of Slovenia National Medical Ethics Committee nr. 132/03/15) and the study followed the statement of the Republic of Slovenia National Medical Ethics Committee nr. 0120-489/2018/7 and principles of the Declaration of Helsinki.

\section{2. Genetic Testing}

Genetic testing was performed at the Clinical Institute for Special Laboratory Diagnostics of the University Children's Hospital at the University Medical Centre Ljubljana. Genomic DNA was isolated from peripheral blood samples with FlexiGene DNA Kit 250 (Qiagen, Hilden, Germany). In male patients, ocular albinism due to GPR143 gene variants was previously excluded. ${ }^{26}$ To evaluate the genetic aetiology of ocular albinism, we performed targeted NGS with TruSightOne Sequencing Panel on the MiSeq platform desktop sequencer coupled with MiSeq Reagent kit v3 (all Illumina, USA). Following on-board primary analysis, we performed secondary data analysis with Variant Studio 2.3 software (Illumina, USA). Rare variants with minor allele frequency less than $5 \%$ in genes reported to be related to syndromic and non-syndromic OCA (AP3B1, BLOC1S3, BLOC1S6, C10ORF11, GPR143, HPS1, HPS3, HPS4, HPS5, HPS6, LYST, MC1R, MITF, MLPH, MYO5A, OCA2, RAB27A, SLC24A5, SLC45A2, TYR, TYRP1) were further evaluated. Possibly causative variants were confirmed by targeted Sanger sequencing using custom oligonucleotides, BigDye Terminator v3.1 sequencing kit, and ABI Genetic Analyser 3500 (both Applied Biosystems, USA).

The pathogenicity of the variants was evaluated as recommended by the American College of Medical Genetics $(\mathrm{ACMG})^{27}$, while novel variants were evaluated with ensemble in silico prediction tools CADD, ${ }^{28}$ REVEL, ${ }^{29}$ VEST $^{30}$ and SpliceAI, ${ }^{31}$ while their frequency in general population was assessed using GnomAd database. ${ }^{32}$ Additionally, we evaluated large deletions and duplications of the OCA2 and TYR genes with multiplex ligation-dependent probe amplification (MLPA). The probe mix SALSA MLPA P325 OCA2 (MRC-Holland, The Netherlands)

Hovnik et al.: Genetic Variability in Slovenian Cohort of Patients ... 
was used according to the manufacturer's instructions. We included three normal control samples to normalize for the allele dosage. We separated amplification products with capillary electrophoresis on ABI Genetic Analyser 3500 (Applied Biosystems, USA) and analysed raw data with GeneMapper ${ }^{\circledast}$ Software Version 4 (Life Technologies, USA). Peak patterns were evaluated using Coffalyser v8 software (MRC-Holland, The Netherlands).

\section{Results}

\section{1. Clinical Characteristics}

Altogether, 25 paediatric individuals, median age 12 years (age range 5-19), 16 male/9 female from 24 un- related families with clinical signs of OCA were included in the study; patients $12^{\star}$ and $13^{\star}$ were brothers. Among them, 16 had normal complexion in regard to the family members. Their clinical characteristics were summarised in the Table 1. All patients had horizontal and/or rotary nystagmus. Patients had no clear deficit of near and colour vision, their corrected vision for distance was within limits of mild amblyopia. The patients' best corrected visual acuity for distance was $0.2-0.6$ Snellen equivalent (0.7-0.2 log MAR). Perimetry revealed no evident lesions of the visual pathways. Electrophysiological evaluation (VEP) showed contralateral asymmetry in all studied children, but not being apparent in the control (Figure 1). Retinal pigmentation was normal or with rare retinal pigment epithelial pigmentation at the posterior pole or retinal vessels, and

Table 1: Clinical finding in children with suspected OCA. M-male, F-female. Iris translucency: - not present; $1 / 3$ one third; $2 / 3$ two thirds; $3 / 3$ total. Foveal pit : \pm underdeveloped; + minimal foveal depression; ++ absent; +++ no foveal depression, choroidal vessels seen at the posterior pole and foveal area. Retinal pigmentepithelial pigmentation: - changes not evident; \pm rare, abnormal periphery; rare + , choroidal vessels seen at the posterior pole, but not in the foveal area; rare ++, choroidal vessels seen also in the foveal area. Refraction: HA - hypermetropic astigmatism, low ( $\leq$ 2.50 D, Dcyl); medium (> 2.5 D, Dcyl); high myopia (-10 and -11 D). Photophobia: + minimal outside; ++ in light condition outside, inside; +++ in normal light, ${ }^{*}$ siblings.

\begin{tabular}{|c|c|c|c|c|c|c|c|}
\hline $\begin{array}{l}\text { Pro- } \\
\text { band }\end{array}$ & Gender & $\begin{array}{l}\text { Skin pigmentation } \\
\text { in the context } \\
\text { of the family }\end{array}$ & $\begin{array}{l}\text { n Iris } \\
\text { translucency }\end{array}$ & $\begin{array}{c}\text { Foveal } \\
\text { pit }\end{array}$ & $\begin{array}{c}\text { Retinal } \\
\text { pigment } \\
\text { epithelial } \\
\text { pigmentation }\end{array}$ & Refraction & $\begin{array}{c}\text { Photop- } \\
\text { hobia }\end{array}$ \\
\hline
\end{tabular}

\begin{tabular}{lllllllll}
\hline 1 & M & unremarkable & $1 / 3$ & + & - & Low HA & - & OCA1 \\
2 & F & unremarkable & $1 / 3$ & \pm & - & Low HA & - & - \\
3 & M & unremarkable & $1 / 2$ & + & - & Low HA & + & Medium HA \\
4 & M & unremarkable & $1 / 3$ & + & Rare + & Low HA & - & - \\
5 & F & unremarkable & $1 / 3$ & + & Rare + & Low HA & - \\
6 & F & unremarkable & $1 / 3$ & + & Rare + & Redium HA & ++ & Highly likely \\
\hline 7 & M & fair & $2 / 3$ & ++ & Rare + & OCA1
\end{tabular}

\begin{tabular}{lllllll}
8 & F & unremarkable & - & + & Rare + & Low HA \\
9 & F & unremarkable & - & Rare + & Low HA \\
\hline 10 & M & unremarkable & $1 / 3$ & +
\end{tabular}

\begin{tabular}{|c|c|c|c|c|c|c|c|c|}
\hline 10 & M & unremarkable & $1 / 3$ & + & Rare + & Low HA & - & \\
\hline 11 & M & fair & $1 / 3$ & + & - & Medium HA & - & \\
\hline $12^{*}$ & M & fair & - & + & Rare + & Low HA & - & \\
\hline $13^{*}$ & M & fair & $1 / 3$ & + & Rare + & Low HA & - & \\
\hline 14 & $\mathrm{~F}$ & unremarkable & $1 / 3$ & + & Rare + & Medium HA & - & \\
\hline 15 & M & fair & - & ++ & Rare + & Medium HA & - & \\
\hline 16 & $\mathrm{~F}$ & unremarkable & $1 / 3$ & + & Rare + & Medium HA & + & OCA3 \\
\hline 17 & M & fair & - & + & Rare + & Medium HA & - & \\
\hline 18 & $\mathrm{M}$ & fair & $2 / 3$ & ++ & Rare + & Medium HA & - & $\mathrm{OCA} 4$ \\
\hline 19 & M & fair & $3 / 3$ & + & Rare + & Medium HA & + & \\
\hline 20 & $\mathrm{~F}$ & fair & $1 / 3$ & +++ & Rare ++ & Low HA & + & $\begin{array}{l}\text { Hermansky- } \\
\text { Pudlak syndrome } \\
\text { type } 1\end{array}$ \\
\hline
\end{tabular}

\begin{tabular}{lcccccc}
\hline 21 & M & unremarkable & $1 / 3$ & + & Rare \pm & Low HA \\
22 & M & unremarkable & - & - & Rare + & Medium HA \\
23 & M & unremarkable & $1 / 3$ & +++ & Rare + & High myopia \\
24 & F & unremarkable & $1 / 3$ & \pm & Rare \pm & Low HA. \\
25 & $\mathrm{M}$ & unremarkable & $1 / 3$ & + & - & Low HA \\
\hline
\end{tabular}


Control child

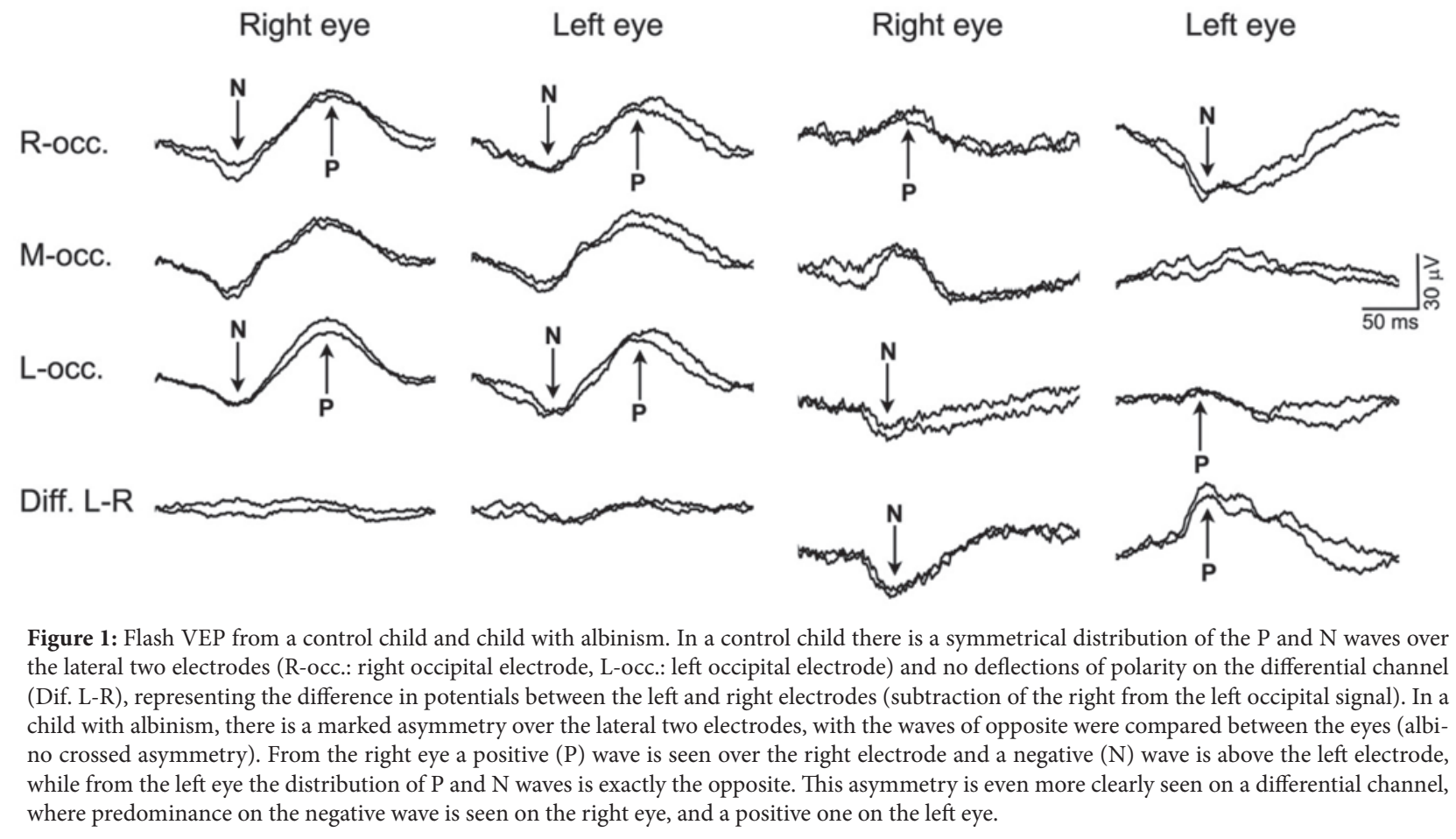

evident macular hypoplasia, but normal optic discs. Complexion, hair and lashes pigmentation did not differ among individual participants. None of the children had really dark or red complexion or hair, iris pigmentation was blue to green, none of the included children had brown iris.

At the time of the first referral to the genetic testing, no additional signs or symptoms were reported. However, at the time when the genetic results were issued in 2018 and results led to the diagnosis of the Hermansky-Pudlak syndrome type 1 , patient 20 she was already referred to the gastroenterological assessment by her paediatrician because of the chronic abdominal pain and occasional diarrhoea. Fulminant Crohn's disease with perianal fistulas was diagnosed and treated with infliximab (Remicade). According to the clinical practice, she was also referred to the paediatric haematology department where prolonged bleeding due to thrombocytopathy was recognised.

\section{2. Genetic Testing}

Genetic characteristics of the cohort were summarised in Table 2. Patients 1 to 6 had pathogenic variants on both alleles of the TYR gene causative for OCA1. Additionally, probands 7 to 15 had variants on both alleles of the TYR gene highly suspected to be causative for at least mild form of OCA1. Patients 16 and 17 had pathogenic variants on both alleles of the TYRP1 gene causative for OCA3. Patients 18 and 19 had pathogenic variants on both alleles of the SLC45A2 gene causative for OCA4, while patient 20 had pathogenic variants on both alleles of the HPS1 gene causative for Hermansky-Pudlak syndrome type 1 . Patients 21 to 25 carried monoallelic variants that could not fully explain the clinical presentations. Parental analysis confirmed the segregation of the detected pathological variants.

Among the genetic variants detected in the cohort, three variants, namely TYR NM_000372.4: c.1430G >A, p.Trp477Ter; SLC45A2 NM_016180.4: c.302G>A, p.Arg101His; TYRP1 NM_000550.2: c.913+1G >A were not previously reported in patients with OCA and were predicted to be deleterious. Their general population data and in silico prediction scores are summarised in the Table 3. SLC45A2 missense variant NM_016180.4: c.302G>A; p.Arg101His was detected in homozygous state in patient 19. Nonsense variant TYR NM_000372.4: c.1430G>A; p.Trp477Ter introducing premature termination codon was detected in patients 3 and 10 in compound heterozygous state with another monoallelic variant in TYR gene. Intronic TYRP1 variant located in consensus splice donor site (TYRP1, NM_000550.2: c.913+1G>A) was detected in patient 21 in heterozygous state, while variant on the other TYRP1 allele has not been detected.

Diagnostic yield after the targeted NGS sequencing was $80 \%$ (20/25). MLPA analysis did not reveal large deletions or duplications in analysed regions of OCA2 or TYR genes, as detected height ratios of the fluorescent peaks were in the normal height ratio range between $0.7-1.3$. 
Table 2: Genetic variants detected in OCA patients (novel variants are in bold; M: male; F: female; homo: homozygous variant; hetero: heterozygous variant; PA-paternal allele, MA-maternal allele, ? segregation analysis was inconclusive; ${ }^{*}$ siblings.).

\begin{tabular}{|c|c|c|c|c|c|}
\hline \multicolumn{2}{|c|}{ Proband } & \multirow{2}{*}{\begin{tabular}{|l} 
Homo \\
Homo
\end{tabular}} & \multirow{2}{*}{$\begin{array}{l}\begin{array}{l}\text { ACMG } \\
\text { classifi- } \\
\text { cation }^{27}\end{array} \\
\begin{array}{l}\text { Likely path. } \\
\text { Benign }\end{array}\end{array}$} & \multirow{2}{*}{$\begin{array}{l}\text { HGMD } \\
\text { Professional } \\
\mathbf{2 0 2 1 . 1}^{\mathbf{3 1}} \\
\text { OCA1, CM981972 } \\
\text { OCA1, CM971555 }\end{array}$} & \multirow{2}{*}{$\begin{array}{l}\text { ClinVar }^{33} \\
\text { Pathogenic } \\
\text { Conflic. int. } \\
\text { path }\end{array}$} \\
\hline $1 / \mathrm{M}$ & $\begin{array}{l}\text { TYR NM_000372.4: c.1A>G (NP_000363.1: p.Met1?) } \\
\text { TYR NM_000372.4: c.1205G>A (NP_000363.1: p.Arg402Gln) }\end{array}$ & & & & \\
\hline $2 / \mathrm{F}$ & $\begin{array}{l}\text { TYR NM_000372.4: c.650G>A (NP_000363.1: p.Arg217Gln) } \\
\text { TYR NM_000372.4: c.1205G>A (NP_000363.1: p.Arg402Gln) }\end{array}$ & $\begin{array}{l}\text { Homo } \\
\text { Homo }\end{array}$ & $\begin{array}{l}\text { Path. } \\
\text { Benign }\end{array}$ & $\begin{array}{l}\text { OCA1, CM930714 } \\
\text { OCA1, CM971555 }\end{array}$ & $\begin{array}{l}\text { Likely path. } \\
\text { Conflic. int. } \\
\text { path }\end{array}$ \\
\hline $3 / \mathrm{M}$ & $\begin{array}{l}\text { TYR NM_000372.4: c.650G>A (NP_000363.1p.Arg217Gln) } \\
\text { TYR NM_000372.4: c.1430G>A (NP_000363.1p.Trp477Ter) }\end{array}$ & $\begin{array}{l}\text { Het,MA } \\
\text { Het,PA }\end{array}$ & $\begin{array}{l}\text { Path. } \\
\text { Likely path. }\end{array}$ & $\begin{array}{l}\text { OCA1, CM930714 } \\
\text { Not reported }\end{array}$ & $\begin{array}{l}\text { Likely path. } \\
\text { Not reported }\end{array}$ \\
\hline $4 / \mathrm{M}$ & $\begin{array}{l}\text { TYR NM_000372.4: c.265T>C (NP_000363.1: p.Cys89Arg) } \\
\text { TYR NM_000372.4: c.1352A>G (NP_000363.1: p.Tyr451Cys } \\
\text { TYR NM_000372.4: c.1217C>T (NP_000363.1: p.Pro406Leu }\end{array}$ & $\begin{array}{l}\text { Het MA } \\
\text { Het PA } \\
\text { Het MA }\end{array}$ & $\begin{array}{l}\text { Path. } \\
\text { Likely path. } \\
\text { Likely path. }\end{array}$ & $\begin{array}{l}\text { OCA1, CM910381 } \\
\text { OCA1, CM117403 } \\
\text { OCA1,CM910385 }\end{array}$ & $\begin{array}{l}\text { Pathogenic } \\
\text { Likely path. } \\
\text { Likely path. }\end{array}$ \\
\hline $5 / \mathrm{F}$ & $\begin{array}{l}\text { TYR NM_000372.4: c.265T>C; (NP_000363.1: p.Cys89Arg) } \\
\text { TYR NM_000372.4: c.325G>A (NP_000363.1: p.Gly109Arg) } \\
\text { TYR NM_000372.4: c.1352A>G (NP_000363.1: p.Tyr451Cys) }\end{array}$ & $\begin{array}{l}\text { Het } \\
\text { Het } \\
\text { Het }\end{array}$ & $\begin{array}{l}\text { Path. } \\
\text { Likely path. } \\
\text { Likely path. }\end{array}$ & $\begin{array}{l}\text { OCA1, CM910381 } \\
\text { OCA1, CM13052 } \\
\text { OCA, CM117403 }\end{array}$ & $\begin{array}{l}\text { Pathogenic } \\
\text { Likely path. } \\
\text { Likely path. }\end{array}$ \\
\hline $6 / F$ & $\begin{array}{l}\text { TYR NM_000372.4: c.1A>G (NP_000363.1: p.Met1?) } \\
\text { TYR NM_000372.4: c.1217C>T (NP_000363.1: p.Pro406Leu) } \\
\text { TYR NM_000372.4: c.1205G>A (NP_000363.1: p.Arg402Gln) } \\
\text { TYR NM_000372.4: c.575C>A (NP_000363.1: p.Ser192Tyr) }\end{array}$ & $\begin{array}{l}\text { Het PA } \\
\text { Het MA } \\
\text { Het PA } \\
\text { Het MA }\end{array}$ & $\begin{array}{l}\text { Likely path. } \\
\text { Likely path. } \\
\text { Benign } \\
\text { Benign }\end{array}$ & $\begin{array}{l}\text { OCA1, CM981972 } \\
\text { OCA1, CM910385 } \\
\text { OCA1, CM971555 }\end{array}$ & $\begin{array}{l}\text { Pathogenic } \\
\text { Likely path. } \\
\text { Conflic. int. } \\
\text { Path } \\
\text { Benign }\end{array}$ \\
\hline $7 / \mathrm{M}$ & $\begin{array}{l}\text { TYR NM_000372.4: c.265T>C (NP_000363.1: p.Cys89Arg) } \\
\text { TYR NM_000372.4: c.1352A>G (NP_000363.1: p.Tyr451Cys) } \\
\text { TYR NM_000372.4: c.1205G>A (NP_000363.1: p.Arg402Gln) } \\
\text { TYR NM_000372.4: c.575C>A (NP_000363.1: p.Ser192Tyr) }\end{array}$ & $\begin{array}{l}\text { Het MA } \\
\text { Het MA } \\
\text { Het PA } \\
\text { Het (?) }\end{array}$ & $\begin{array}{l}\text { Path. } \\
\text { Likely path. } \\
\text { Benign } \\
\text { Benign }\end{array}$ & $\begin{array}{l}\text { OCA1, CM910381 } \\
\text { OCA, CM117403 } \\
\text { OCA1, CM971555 }\end{array}$ & $\begin{array}{l}\text { Path } \\
\text { Likely path. } \\
\text { Conflic. int. } \\
\text { path. } \\
\text { Benign }\end{array}$ \\
\hline $8 / \mathrm{F}$ & $\begin{array}{l}\text { TYR NM_000372.4: c.1063G >C (NP_000363.1: p. Ala355Pro) } \\
\text { TYR NM_000372.4: c.1217C>T (NP_000363.1: p.Pro406Leu) } \\
\text { TYR NM_000372.4: c.1205G>A (NP_000363.1: p.Arg402Gln) } \\
\text { TYR NM_000372.4: c.575C>A (NP_000363.1: p.Ser192Tyr) }\end{array}$ & $\begin{array}{l}\text { Het PA } \\
\text { Het PA } \\
\text { Het MA } \\
\text { Het MA }\end{array}$ & $\begin{array}{l}\text { Path. } \\
\text { Likely path } \\
\text { Benign } \\
\text { Benign }\end{array}$ & $\begin{array}{l}\text { OCA1, CM971550 } \\
\text { OCA1, CM910385 } \\
\text { OCA1, CM971555 }\end{array}$ & $\begin{array}{l}\text { Pathogenic } \\
\text { Likely path. } \\
\text { Conflic. int. } \\
\text { path. } \\
\text { Benign }\end{array}$ \\
\hline path & $\begin{array}{l}\text { TYR NM_000372.4: c.265T>C; (NP_000363.1: p.Cys89Arg) } \\
\text { TYR NM_000372.4: c.1352A>G (NP_000363.1: p.Tyr451Cys) } \\
\text { TYR NM_000372.4: c.1205G>A (NP_000363.1: p.Arg402Gln) }\end{array}$ & $\begin{array}{l}\text { Het PA } \\
\text { Het PA } \\
\text { Het MA }\end{array}$ & $\begin{array}{l}\text { Path. } \\
\text { Likely path. } \\
\text { Benign }\end{array}$ & $\begin{array}{l}\text { OCA1, CM910381 } \\
\text { OCA, CM117403 } \\
\text { OCA1, CM971555 }\end{array}$ & $\begin{array}{l}\text { Pathogenic } \\
\text { Likely path. } \\
\text { Conflic. int. }\end{array}$ \\
\hline $10 / \mathrm{M}$ & $\begin{array}{l}\text { TYR NM_000372.4: c.1430G>A (NP_000363.1p.Trp477Ter) } \\
\text { TYR NM_000372.4: c.1205G>A (NP_000363.1: p.Arg402Gln) } \\
\text { TYR NM_000372.4: c.575C>A (NP_000363.1: p.Ser192Tyr) }\end{array}$ & $\begin{array}{l}\text { Het PA } \\
\text { Het MA } \\
\text { Het (?) }\end{array}$ & $\begin{array}{l}\text { Likely path. } \\
\text { Benign. } \\
\text { Benign }\end{array}$ & $\begin{array}{l}\text { Not reported } \\
\text { OCA1, CM971555 }\end{array}$ & $\begin{array}{l}\text { Not reported } \\
\text { Conflic. int. } \\
\text { Path } \\
\text { Benign }\end{array}$ \\
\hline $11 / \mathrm{M}$ & $\begin{array}{l}\text { TYR NM_000372.4: c.650G>A (NP_000363.1: p.Arg217Gln) } \\
\text { TYR NM_000372.4: c.1205G>A (NP_000363.1: p.Arg402Gln) }\end{array}$ & $\begin{array}{l}\text { Het } \\
\text { Homo }\end{array}$ & $\begin{array}{l}\text { Path. } \\
\text { Benign }\end{array}$ & $\begin{array}{l}\text { CM930714 } \\
\text { CM041478 }\end{array}$ & $\begin{array}{l}\text { Likely path. } \\
\text { Conflic. int. } \\
\text { path }\end{array}$ \\
\hline $12^{*} / \mathrm{M}$ & $\begin{array}{l}\text { TYR NM_000372.4: c.650G>A (NP_000363.1: p.Arg217Gln) } \\
\text { TYR NM_000372.4: c.1205G>A (NP_000363.1: p.Arg402Gln) } \\
\text { TYR NM_000372.4: c.575C>A (NP_000363.1: p.Ser192Tyr) }\end{array}$ & $\begin{array}{l}\text { Het } \\
\text { Homo } \\
\text { Het }\end{array}$ & $\begin{array}{l}\text { Path. } \\
\text { Benign } \\
\text { Benign }\end{array}$ & $\begin{array}{l}\text { OCA1, CM930714 } \\
\text { OCA1, CM971555 }\end{array}$ & $\begin{array}{l}\text { Likely path. } \\
\text { Conflic. int. } \\
\text { Path } \\
\text { Benign }\end{array}$ \\
\hline $13^{*} / \mathrm{M}$ & $\begin{array}{l}\text { TYR NM_000372.4: c.650G>A (NP_000363.1: p.Arg217Gln) } \\
\text { TYR NM_000372.4: c.1205G>A (NP_000363.1: p.Arg402Gln }\end{array}$ & $\begin{array}{l}\text { Het } \\
\text { Homo }\end{array}$ & $\begin{array}{l}\text { Path. } \\
\text { Benign }\end{array}$ & $\begin{array}{l}\text { OCA1, CM930714 } \\
\text { OCA1, CM971555 }\end{array}$ & $\begin{array}{l}\text { Likely path. } \\
\text { Conflic. int. } \\
\text { Path } \\
\text { Benign }\end{array}$ \\
\hline
\end{tabular}




\begin{tabular}{|c|c|c|c|c|c|}
\hline Proban & Variant & & ACMG & HGMD & ClinVar 33 \\
\hline $14 / \mathrm{F}$ & TYR NM_000372.4: c1217C>T (NP_000363.1: p.Pro406Leu) & Het PA & Likely path & OCA1, CM910385 & Likely path. \\
\hline & TYR NM_000372.4: c.1205G>A (NP_000363.1: p.Arg402Gln) & Het MA & Benign & OCA1, CM971555 & $\begin{array}{l}\text { Conflic. int. } \\
\text { Path }\end{array}$ \\
\hline & TYR NM_000372.4: c.575C>A (NP_000363.1: p.Ser192Tyr) & Homo & Benign & & Benign \\
\hline $15 / \mathrm{M}$ & TYR NM_000372.4: c.1217C>T (NP_000363.1: p.Pro406Leu) & Het & Likely path & OCA1, CM910385 & Likely path. \\
\hline & TYR NM_000372.4: c.1205G>A (NP_000363.1: p.Arg402Gln) & Het & Benign & OCA1, CM971555 & $\begin{array}{l}\text { Conflic. int. } \\
\text { Path }\end{array}$ \\
\hline & TYR NM_000372.4: c.575C>A (NP_000363.1: p.Ser192Tyr) & Homo & Benign & & Benign \\
\hline $16 / \mathrm{F}$ & TYRP1, NM_000550.2: c.670C>T (NP_000541.1:p.His224Tyr) & Homo & Likely path. & Not reported & Likely path. \\
\hline $17 / \mathrm{M}$ & TYRP1 NM_000550.2: c.70G>A (NP_000541.1: p.Ala24Thr & Het & Benign & OCA3: & $\begin{array}{l}\text { Conflic. int. } \\
\text { path }\end{array}$ \\
\hline & TYRP1 NM_000550.2: c.418G>T (NP_000541.1:p.Glu140Ter) & Het & Pathogenic & $\begin{array}{l}\text { CM135782 } \\
\text { OCA3: } \\
\text { CM172531 }\end{array}$ & Not reported \\
\hline $18 / \mathrm{M}$ & $\begin{array}{l}\text { SLC45A2 NM_016180.4: c.606G >C } \\
\text { (NP_057264.3:p.Trp202Cys) }\end{array}$ & Homo & Likely path. & OCA4: CM040231 & $\begin{array}{l}\text { Conflic. int. } \\
\text { Path/ Likely } \\
\text { path. }\end{array}$ \\
\hline $19 / \mathrm{M}$ & $\begin{array}{l}\text { SLC45A2 NM_016180.4: c.302G }>\text { A } \\
\text { (NP_057264.3:p. Arg101His) }\end{array}$ & Homo & Likely path. & Not reported & Not reported \\
\hline & TYR NM_000372.4: c. 589G>A (NP_000363.1:p.Asp197Asn) & Het & VUS & Not reported & Not reported \\
\hline $20 / \mathrm{F}$ & OCA2 NM_000275.2: c.1025A>G（p.Tyr342Cys） & Het & Likely path. & $\begin{array}{l}\text { OCA, } \\
\text { CM091279 }\end{array}$ & $\begin{array}{l}\text { Likely path./ } \\
\text { VUS }\end{array}$ \\
\hline & $\begin{array}{l}\text { HPS1, NM_000195.3: c.972dupC } \\
\text { (NP_000186.2:p.Met325HisfsTer128) }\end{array}$ & Het & Pathogenic & $\begin{array}{l}\text { Hermansky- } \\
\text { Pudlak, CI962292 }\end{array}$ & Likely path \\
\hline & $\begin{array}{l}\text { HPS1, NM_000195.3: c 1189delC } \\
\text { (NP_000186.2:p.Gln397SerfsTer2) }\end{array}$ & Het & Pathogenic & $\begin{array}{l}\text { Hermansky- } \\
\text { Pudlak, CD982692 }\end{array}$ & Likely path \\
\hline $21 / \mathrm{M}$ & TYRP1, NM_000550.2: c.913+1G >A & Het & Likely path. & Not reported & Not reported \\
\hline $22 / \mathrm{M}$ & TYR NM_000372.4: c.1205G>A (NP_000363.1p.Arg402Gln) & Het & Benign & $\begin{array}{l}\text { OCA1, } \\
\text { CM971555 }\end{array}$ & $\begin{array}{l}\text { Conflic. int. } \\
\text { path }\end{array}$ \\
\hline & TYR NM_000372.4: c.575C>A (NP_000363.1: p.Ser192Tyr) & Homo & Benign & & Benign \\
\hline $23 / \mathrm{M}$ & TYR NM_000372.4: c.1205G>A (NP_000363.1p.Arg402Gln) & Het & Benign & $\begin{array}{l}\text { OCA1, } \\
\text { CM971555 }\end{array}$ & $\begin{array}{l}\text { Conflic. int. } \\
\text { path }\end{array}$ \\
\hline $24 / \mathrm{F}$ & TYR NM_000372.4: c.575C>A (NP_000363.1: p.Ser192Tyr) & Het & Benign & & Benign \\
\hline $25 / \mathrm{M}$ & TYR NM_000372.4: c.575C>A (NP_000363.1: p.Ser192Tyr) & Homo & Benign & & Benign \\
\hline
\end{tabular}

Table 3: General population frequencies and in silico prediction scores of the novel genetic variants detected in OCA patients (REVEL ${ }^{29}$, VEST4 ${ }^{30}$ and SpliceAI ${ }^{31}$ values $\mathrm{O}-1$, and $\mathrm{CADD}^{28}$ Phred values: variants with higher scores are predicted to be more likely pathogenic; nd: not detected)

\begin{tabular}{lll}
\hline Variant & $\begin{array}{l}\text { Position and MAF } \\
\left(\text { gnomAD }{ }^{32}\right)\end{array}$ & $\begin{array}{l}\text { In silico prediction tools } \\
\text { and scores }\end{array}$ \\
\hline TYR NM_000372.4: c.1430G>A (NP_000363.1p.Trp477Ter) & nd & $\begin{array}{l}\text { CADD: } 39 \\
\text { VEST4:0,88 }\end{array}$ \\
\hline SLC45A2 NM_016180.4: c.302G>A (NP_057264.3:p. Arg101His) & $\begin{array}{l}\text { chr5:33984282: rs763531791 } \\
\text { CAF: A }=0,0024 \%\end{array}$ & $\begin{array}{l}\text { CADD: } 36,6 \\
\text { REVEL:0.829 } \\
\text { VEST4: } 0.65\end{array}$ \\
\hline TYRP1, NM_000550.2: c.913+1G>A & $\begin{array}{l}\text { chr9:1251,262 alleles) } \\
\text { MAF: A }=0,0016 \%\end{array}$ & CADD: 34 \\
& $(4 / 250,176$ alleles $)$ & SpliceAI: 0.85
\end{tabular}




\section{Discussion}

OCA shows considerable clinical heterogeneity. ${ }^{2}$ Consequently, individual types of albinism might be difficult to differentiate clinically in children, especially in those with light complexion, where there might be an overlap with other related disorders. Even though OCA is a genetically heterogeneous disorder, NGS based genetic testing enables timely definitive etiological diagnosis and consequently appropriate management of the disease. This is of notable importance in cases with mild or partial clinical manifestations. ${ }^{10}$

Among 25 paediatric patients of Slovenian descent with clinically suspected OCA, as much as 16 had normal complexion in regard to the other family members, and some of them had only mild albinism-related features (Table 1$)$. In as much as 20 out of 25 patients $(80 \%)$ in the entire cohort, genetic variants explaining their clinical phenotype were identified (Table 2). This is in accordance with other recent reports, where molecular diagnosis was achieved in $92 \%$ of patients with mild partial albinism ${ }^{10}$ and $72,3 \%$ of patients with albinism. ${ }^{12}$ Great majority of patients in our cohort $(60 \%-15 / 25)$ had TYR disease causing variants. This is surprisingly high when compared to the large cohort of 990 tested patients in France, where the number was $41,8 \%{ }^{12}$. High frequency of $T Y R$ disease causing variants can partly be explained with the fact that ocular albinism due to GPR 143 variants was previously excluded from our group of patients. ${ }^{26}$ For instance in above mentioned large cohort GPR143 variants were responsible for $7 \%$ of the cases. ${ }^{12}$ Among our remaining patients, $4 \%$ $(1 / 25)$ had only monoallelic disease causing variants that cannot fully explain the clinical presentation, while in $16 \%$ $(4 / 25)$ no disease causing variant was detected in analysed genes. This is comparable with the large reported cohort, where $12 \%$ of patients had monoallelic variants and $15,5 \%$ had no detected disease causing variants. ${ }^{12}$ Surprisingly, we did not identify variants in $O C A 2$ gene that would be a probable cause of albinism in our cohort. We identify only one OCA 2 variant in heterozygous state in a patient 20 carrying HPS1 disease causing variants. NGS results in this study were confirming the results of our earlier testing approach using selective gene Sanger sequencing. This is in concordance with a previously reported group of patients with partial OCA, where OCA2 disease causing variants were identified only in heterozygous state together with the heterozygous variant in TYR gene. ${ }^{10}$

The novel TYR NM_000372.4: $\quad$ c.1430G $>$ A (NP_000363.1 p.Trp477Ter) variant was detected in patients 3 and 10 in compound heterozygous state with another TYR variant. This variant is introducing premature termination codon and was predicted to be pathogenic. Both patients harbouring this variant had mild signs of OCA with normal complexion, mild to medium iris transillumination, foveal depression still present, but smaller, normal or rare retinal pigment epithelium, and no photo- phobia. The mild phenotypic characteristics in patient 10 are likely associated with hypomorfic variant present on the other TYR gene allele as it was previously reported. ${ }^{14}$

SLC45A2 variant NM_016180.4: c.302G $>$ A (NP_057264.3: p.Arg101His) was detected in homozygous state in patient 19. It has so far not been reported in OCA and was predicted to be pathogenic. A different amino acid change, namely p.Arg101Cys, on the same position as here reported variant, was previously reported in a patient with OCA4, but his clinical characteristics were not described in more details (HGMD acc. nr. CM083847). Variants in SLC45A2 gene are associated with OCA4. This is a rare OCA type, reported in approximately $3 \%$ of European patients, ${ }^{34-36}$ but is more common in Japan..$^{37}$ OCA4 is clinically variable and overlapping with other OCA types. ${ }^{5}$ Patient 19 had mild clinical phenotype with fair complexion, minimal foveal depression, rare retinal pigment epithelium, choroidal vessels seen at the posterior pole, but not in the foveal area, medium hypermetropic astigmatism, and minimal photophobia. His clinical presentation did not differ significantly from other patients, nevertheless, he was the only patients in this group with complete iris transillumination.

Variants in TYRP1 gene are associated with OCA3, an extremely rare type in Caucasian patients, reported only in individual patients ${ }^{17}$ and in $2,1 \%$ of patients in a cohort of mainly but not exclusively French origin. ${ }^{12}$ Patient 16 had homozygous TYRP1 variant NM_000550.2:c.670C $>$ T (NP_000541.1:p.His224Tyr) that was previously reported in ClinVar database in a patient with ocular albinism (Allele ID: 360904) but was so far not reported in Human Gene Mutation Database. He had normal complexion, mild iris transillumination, choroidal vessels seen at the posterior pole, but not in the foveal area, medium hypermetropic astigmatism, and minimal photophobia. His clinical presentation did not differ significantly from other patients and was not concordant with the reported Caucasian patients, who had light-yellow skin, yellow-gold hair with orange highlights and fair eyelashes, divergent strabismus, and no photophobia. ${ }^{17}$

Among the variants that were classified as highly susceptive for OCA was also the TYR gene harbouring two allelic variants, namely NM_000372.4:c.575C >A (p.Ser192Tyr) and NM_000372.4: c.1205G>A (p.Arg402Gln). Those variants individually are frequently reported in general population and consequently regarded as benign. Nevertheless, when these are located in cis they are much rarer and when inherited in trans with pathogenic TYR variant, they were repeatedly reported to be causative for mild or partial form of OCA. ${ }^{10-13}$ This complex TYR allele was present in several patients in our cohort, while in six patients (subjects $8,10,12,13,14,15$ ) it was inherited in trans with other TYR variant. Therefore, those patients we classified as likely OCA1. They all had normal or fair complexion, rare retinal pigment epithelium, low or medium hypermetropic astigmatism, and no photophobia - con-

Hovnik et al.: Genetic Variability in Slovenian Cohort of Patients ... 
cordant with mild OCA. Among them, two were brothers (subject 12 and 13) with very similar clinical presentation. They only differed in iris transillumination that was present in mild form only in the younger brother (subject 13). Similarly as in our cohort, this complex allele was the most common disease causing allele in a previously reported group of patients with partial OCA. ${ }^{10}$

Patient 20 was compound heterozygote for two known disease causing variants in HPS1 gene associated with Hermansky-Pudlak syndrome type 1. Additionally, she was a heterozygous carrier of know disease causing variant in OCA2 gene associated with OCA2. In our cohort, she had the most severe retinal pigment epithelium phenotype with choroidal vessels seen also in the foveal area. She had fair complexion, mild iris transillumination, no foveal depression, low hypermetropic astigmatism, and photophobia. She was referred to genetic testing due to her albinism-related symptoms and the genetic testing results led to the diagnosis of the Hermansky-Pudlak syndrome type 1 in 2018 when she was 11 years old. Later on, fulminant Crohn's disease with perianal fistulas and prolonged bleeding due to thrombocytopathy were recognised, both known manifestations of Hermansky-Pudlak syndrome..$^{38,39}$ The diagnostic pathway that led to the diagnosis of Hermansky-Pudlak syndrome in this patient confirms the importance of genetic testing in children with albinism. As previously suggested, early identification of the genetic aetiology of albinism can reveal serious syndromic causes of albinism that need appropriate clinical management including clinical evaluation of possible extraocular manifestations. ${ }^{40}$

In our cohort of patients, phenotype could not predict genotype or vice versa, and there were no specific clinical signs correlating with the molecular diagnosis. Nevertheless, these children seem to be of lighter complexion with greater foveal hypoplasia and rare retinal pigment epithelium. Iris transillumination did not correlate with photophobia or other clinical signs, but it was present to a certain extent in all subjects. Furthermore, all subjects had low to medium hypermetropic astigmatism, with one who was highly myopic.

In conclusion, NGS based genetic testing had a high diagnostic yield of $80 \%$ in our paediatric cohort of patients with various degrees of OCA and previously excluded OA due to disease causing variants in GPR143 gene. Interestingly, we have identified a patient of white European ancestry with OCA3, which is an extremely rare report, and one patient with OCA due to the Hermansky-Pudlak syndrome type 1 .

\section{Acknowledgements}

The authors thank Assist. Prof. Jelka Brecelj, PhD, and Assist. Prof. Maja Šuštar, PhD, for electrophysiological assessment and assistance, as well as Mrs. Barbara Klemenc for technical assistance with OCTs and fundus imaging.
This work was supported by the Slovenian Research Agency (grants P3-0343, P1-0170).

\section{Declaration of interest:}

There is no conflict of interest declared.

\section{References}

1. Marçon CR, Maia M. Albinism: epidemiology, genetics, cutaneous characterization, psychosocial factors. An Bras Dermatol. 2019; 94(5), 503-520. DOI:10.1016/j.abd.2019.09.023

2. Montoliu L, Grønskov K, Wei AH, et al. Increasing the complexity: New genes and new types of albinism. Pigment Cell Melanoma Res. 2014; 27(1), 11-18.

DOI:10.1111/pcmr.12167

3. Käsmann-Kellner B, Seitz B. Phänotyp des visuellen systems bei okulokutanem und okulärem albinismus. Ophthalmologe. 2007; 104(8), 648-661. DOI:10.1007/s00347-007-1571-4

4. Kruijt CC, de Wit GC, Bergen AA, Florijn RJ, Schalij-Delfos NE, van Genderen MM. The Phenotypic Spectrum of Albinism. Ophthalmology. 2018, 125(12), 1953-1960. DOI:10.1016/j.ophtha.2018.08.003

5. Summers CG. Albinism: Classification, clinical characteristics, and recent findings. Optom Vis Sci. 2009; 86(6), 659662. DOI:10.1097/OPX.0b013e3181a5254c

6. Summers CG, King RA. Ophthalmic Features of Minimal Pigment Oculocutaneous Albinism. Ophthalmology. 1994; 101(5), 906-914. DOI:10.1016/S0161-6420(13)31250-0

7. Spritz R, Strunk K, LB G, RA K. The New England Journal of Medicine Downloaded from nejm.org on April 1, 2015. For personal use only. No other uses without permission. Copyright (c) 1990 Massachusetts Medical Society. All rights reserved. New Englnd J Med. 1990; 322(24), 1724-1728. DOI:10.1056/NEJM199006143222407

8. Lerner AB, Fitzpatrick TB. Biochemistry of melanin formation. Physiol Rev. 1950; 30(1), 91-126.

DOI:10.1152/physrev.1950.30.1.91

9. King RA, Pietsch J, Fryer JP, et al. Tyrosinase gene mutations in oculocutaneous albinism 1 (OCA1): Definition of the phenotype. Hum Genet. 2003; 113(6), 502-513.

DOI:10.1007/s00439-003-0998-1

10. Campbell P, Ellingford JM, Parry NRA, et al. Clinical and genetic variability in children with partial albinism. Sci Rep. 2019; 9(1), 16576. DOI:10.1038/s41598-019-51768-8

11. Norman CS, O'Gorman L, Gibson J, et al. Identification of a functionally significant tri-allelic genotype in the Tyrosinase gene (TYR) causing hypomorphic oculocutaneous albinism (OCA1B). Sci Rep. 2017; 7(1), 4415.

DOI:10.1038/s41598-017-04401-5

12. Lasseaux E, Plaisant C, Michaud V, et al. Molecular characterization of a series of 990 index patients with albinism. Pigment Cell Melanoma Res. 2018; 31(4), 466-474. DOI:10.1111/pcmr.12688

13. Jagirdar K, Smit DJ, Ainger SA, et al. Molecular analysis of 
common polymorphisms within the human Tyrosinase locus and genetic association with pigmentation traits. Pigment Cell Melanoma Res. 2014; 27(4), 552-564.

DOI:10.1111/pcmr.12253

14. Monfermé S, Lasseaux E, Duncombe-Poulet C, et al. Mild form of oculocutaneous albinism type 1: Phenotypic analysis of compound heterozygous patients with the R402Q variant of the TYR gene. Br J Ophthalmol. 2019; 103(9), 1239-1247. DOI:10.1136/bjophthalmol-2018-312729

15. Rooryck C, Morice-Picard F, Lasseaux E, et al. High resolution mapping of OCA2 intragenic rearrangements and identification of a founder effect associated with a deletion in Polish albino patients. Hum Genet. 2011; 129(2), 199-208. DOI:10.1007/s00439-010-0913-5

16. Rosemblat S, Durham-Pierre D, Gardner JM, Nakatsu Y, Brilliant MH, Orlow SJ. Identification of a melanosomal membrane protein encoded by the pink-eyed dilution (type II oculocutaneous albinism) gene. Proc Natl Acad Sci U S A. 1994; 91(25), 12071-12075. DOI:10.1073/pnas.91.25.12071

17. Rooryck C, Roudaut C, Robine E, Müsebeck J, Arveiler B. Oculocutaneous albinism with TYRP1 gene mutations in a Caucasian patient. Pigment Cell Res. 2006; 19(3), 239-242. DOI:10.1111/j.1600-0749.2006.00298.x

18. Newton JM, Cohen-Barak O, Hagiwara N, et al. Mutations in the human orthologue of the mouse underwhite gene (uw) underlie a new form of oculocutaneous albinism, OCA4. Am J Hum Genet. 2001; 69(5), 981-8. DOI:10.1086/324340

19. Bin BH, Bhin J, Yang SH, et al. Membrane-associated transporter protein (MATP) regulates melanosomal $\mathrm{pH}$ and influences tyrosinase activity. PLoS One. 2015; 10(6), e0129273. DOI:10.1371/journal.pone.0129273

20. Pennamen P, Tingaud-Sequeira A, Gazova I, et al. Dopachrome tautomerase variants in patients with oculocutaneous albinism. Genet Med. 2021; 23(3), 479-487. DOI:10.1038/s41436-020-00997-8

21. Gargiulo A, Testa F, Rossi S, et al. Molecular and clinical characterization of albinism in a large cohort of Italian patients. Investig Ophthalmol Vis Sci. 2011.

DOI:10.1167/iovs.10-6091

22. Thomas MG, Kumar A, Mohammad S, et al. Structural grading of foveal hypoplasia using spectral-domain optical coherence tomography: A predictor of visual acuity? Ophthalmology. 2011. DOI:10.1016/j.ophtha.2011.01.028

23. Brecelj J. Visual electrophysiology in the clinical evaluation of optic neuritis, chiasmal tumours, achiasmia, and ocular albinism: an overview. Doc Ophthalmol. 2014; 129(2), 71-84. DOI:10.1007/s10633-014-9448-8

24. Simeonov DR, Wang X, Wang C, et al. DNA Variations in Oculocutaneous Albinism: An Updated Mutation List and Current Outstanding Issues in Molecular Diagnostics. Hum Mutat. 2013; 34(6), 827-835. DOI:10.1002/humu.22315

25. Brecelj J, Sustar M, Pečarič-Meglič N, Škrbec M, Stirn-Kranjc B. VEP characteristics in children with achiasmia, in comparison to albino and healthy children. Doc Ophthalmol. 2012; 124(2), 109-123. DOI:10.1007/s10633-012-9315-4

26. Trebušak Podkrajšek K, Stirn Kranjc B, Hovnik T, Kovač
J, Battelino T. GPR143 gene mutation analysis in pediatric patients with albinism. Ophthalmic Genet. 2012; 33(3), 167170. DOI:10.3109/13816810.2011.559651

27. Richards S, Aziz N, Bale S, et al. Standards and guidelines for the interpretation of sequence variants: A joint consensus recommendation of the American College of Medical Genetics and Genomics and the Association for Molecular Pathology. Genet Med. 2015; 17(5), 405-424.

DOI:10.1038/gim.2015.30

28. Combined Annotation Dependent Depletion. http://cadd. gs.washington.edu/.

29. Ioannidis NM, Rothstein JH, Pejaver V, et al. REVEL: An Ensemble Method for Predicting the Pathogenicity of Rare Missense Variants. Am J Hum Genet. 2016; 99(4) 877-885. DOI:10.1016/j.ajhg.2016.08.016

30. Douville C, Masica DL, Stenson PD, et al. Assessing the Pathogenicity of Insertion and Deletion Variants with the Variant Effect Scoring Tool (VEST-Indel). Hum Mutat. 2016; 37(1) 28-35. DOI:10.1002/humu.22911

31. Jaganathan K, Kyriazopoulou Panagiotopoulou S, McRae JF, et al. Predicting Splicing from Primary Sequence with Deep Learning. Cell. 2019; 176(3), 535-548.e24.

DOI:10.1016/j.cell.2018.12.015

32. The Genome Aggregation Database. http://gnomad.broadinstitute.org//.

33. Landrum MJ, Chitipiralla S, Brown GR, et al. ClinVar: Improvements to accessing data. Nucleic Acids Res. 2020; 48(D1), D835-D844. DOI:10.1093/nar/gkz972

34. Mauri L, Barone L, Al Oum M, et al. SLC45A2 mutation frequency in Oculocutaneous Albinism Italian patients doesn't differ from other European studies. Gene. 2014; 533(1), 398402. DOI:10.1016/j.gene.2013.09.053

35. Grønskov K, Ek J, Sand A, et al. Birth prevalence and mutation spectrum in Danish patients with autosomal recessive albinism. Investig Ophthalmol Vis Sci. 2009; 50(3), 10581064. DOI:10.1167/iovs.08-2639

36. Rundshagen U, Zühlke C, Opitz S, Schwinger E, Käsmann-Kellner B. Mutations in the MATP Gene in Five German Patients Affected by Oculocutaneous Albinism Type 4 . Hum Mutat. 2004; 23(2), 106-110.

DOI:10.1002/humu.10311

37. Inagaki K, Suzuki T, Shimizu H, et al. Oculocutaneous Albinism Type 4 Is One of the Most Common Types of Albinism in Japan. Am J Hum Genet. 2004; 74(3), 466-471. DOI: $10.1086 / 382195$

38. De Jesus Rojas W, Young LR. Hermansky-Pudlak Syndrome. Semin Respir Crit Care Med. 2020; 41(2), 238-246. DOI:10.1055/s-0040-1708088 8

39. El-Chemaly S, Young LR. Hermansky-Pudlak Syndrome. Clin Chest Med. 2016; 37(3), 505-511.

DOI:10.1016/j.ccm.2016.04.012

40. Lenassi E, Clayton-Smith J, Douzgou S, et al. Clinical utility of genetic testing in 201 preschool children with inherited eye disorders. Genet Med. 2020; 22(4), 745-751.

DOI:10.1038/s41436-019-0722-8 


\section{Povzetek}

Okulokutani albinizem (OCA) je dedna motnja, ki vpliva na vidni sistem in pigmentacijo kože. Naš cilj je bil z uporabo naprednega molekularno-genetskega pristopa oceniti genetsko in klinično heterogenost v kohorti slovenskih pediatričnih pacientov s klinično domnevanim OCA. Pri 20 od 25 pacientov so bile ugotovljene genetske različice, ki pojasnjujejo njihov klinični fenotip. Velika večina pacientov (15/25) je imela genetske različice gena TYR, povezanega $\mathrm{z}$ OCA tipa 1, sledile so mu različice genov TYRP1, SLC45A2 in HPS1, ki so vzrok za OCA3, OCA4 in Hermansky-Pudlakov sindrom tipa 1. Ugotovili smo, da fenotip OCA ne more napovedati genotipa in obratno. Kljub temu je bil diagnostični izkoristek po ciljanem sekvenciranju naslednje generacije (NGS) $80 \%$ in se je izkazal za učinkovitega v naši pediatrični kohorti pacientov z različno stopnjo OCA. Tudi pri 16 pacientih z normalno poltjo je bil diagnostični izkoristek $62,5 \%$. Zanimivo je, da smo identificirali pacienta belega evropskega porekla z OCA3, kar je izjemno redko poročano, in enega pacienta z OCA zaradi Hermansky-Pudlakovega sindroma tipa 1. 